\title{
LA RESILIENCIA COMO HERRAMIENTA DE PROTECCIÓN FAMILIAR
}

\author{
Leidy Daian González Arias y Diana Patricia Landazábal Cuervo ${ }^{87}$
}

RESUMEN

Esta ponencia presenta los resultados de una investigación que analizaba la construcción colectiva de estrategias de protección, fortalecimiento de la resiliencia y disminución del riesgo social en familias de la FNSA (Fundación Nuestra Señora del Amor) de la localidad Ciudad Bolívar en Bogotá. La metodología utilizada fue de enfoque cualitativo y tipo participativo con una muestra de 10 familias cuyos miembros oscilan entre los 0 y 60 años, población total 45 personas. Las técnicas de recolección, fueron observación participante, historias de vida y entrevista semiestructurada. Se diagnosticaron factores resilientes, de riesgo y funcionalidad familiar, que permitieron el diseño de estrategias y posteriormente una evaluación que indicaba la relación de la resiliencia como herramienta creativa para mejorar la calidad de vida de los participantes. Los resultados mostraron que al implementar la resiliencia disminuyó el riesgo de ruptura del sistema familiar, fortaleciendo pautas de crianza, relaciones interpersonales y valores, además de incrementar la expresión del afecto y aspiraciones, como factores protectores para la reconstrucción del vínculo familiar.

Palabras clave: familias, factores resilientes, riesgo, ruptura familiar, vulnerabilidad, estrategias de fortalecimiento y trabajo en red.

\begin{abstract}
This communication presents the results of an investigation that analyzed the collective construction of strategies of protection, fortification of the resilience and diminution of the social risk in families of the FNSA of the locality City Bolivar in Bogota. The used methodology was of qualitative approach and participating type with a sample of 10 families in ages between 0 to 60 years, total population 45 people. The harvesting techniques were participant observation, histories of life and semi structured interview. Resilience factors were diagnosed, of risk and familiar functionality, that later allowed to the design of strategies and an evaluation that indicated the relation of the resilience like creative tool to improve the quality of life of the participants. The results showed that when implementing the resilience diminished the risk of rupture of the familiar system, fortifying you rule of interpersonal raising, relations and values, besides increasing the expression of the affection and aspirations, like protective factors for the reconstruction of the familiar bond.
\end{abstract}

Key words: Resilientes Families, Factors, Risk, Familiar Rupture, Vulnerability, Strategies of Fortification and Work in Network.

\footnotetext{
87 Grupo de Investigación: Gestión Vital, Código de registro del grupo en Colciencias: COL0056645, Nombre del Semillero: Fénix, Ave de Luz, Línea de Investigación: Educación y desarrollo humano. Escuela de Ciencias de la Educación. Red de Investigación: Desarrollo Humano, Convivencia y productividad. Correo: diana.landazabal@unad.edu.co
} 


\section{INTRODUCCIÓN}

Esta ponencia da una mirada frente a las acciones que se trabajaron en una investigación aplicada a población familiar en situación de vulnerabilidad a través de los factores resilientes, con el interés de vincular el proceso de resiliencia al manejo de problemáticas como el desplazamiento, la pobreza y demás situaciones que afectan a las familias bogotanas. Muchos de estos problemas individuales se fundan en el núcleo familiar, tomando a la familia como unidad básica de la sociedad, en la que se consolidan los procesos biológicos, sociales, económicos, políticos y espirituales, se vive una etapa larga de la vida, adquiriendo valores y bases para la construcción de lo que se es o se llegará a ser, comprendiendo que los cambios se deben emprender en el hogar.

El problema de investigación se basó en el análisis de la situación al interior de la familia, y se buscó medir el riesgo en el que se encuentran las familias de una localidad de Bogotá e intervenir para mejorar sus problemáticas. Este trabajo buscaba entender metodologías para el fortalecimiento de la resiliencia en la disminución del riesgo en las familias de la Fundación, constituyéndose en un problema de investigación, que permitió reconocer a la población y reincidir en el desarrollo de capacidades y en la solución de las dificultades presentes por estos factores antes mencionados. La pregunta que se formuló fue: ¿Cuál es el efecto de aplicar estrategias para el uso y fortalecimiento de la resiliencia, en la disminución del riesgo de ruptura del sistema familiar en la comunidad de la FNSA, ubicada en la localidad Ciudad Bolívar?

El objetivo de la investigación fue reflexionar en torno a la fundamentación de estrategias de fortalecimiento de los factores resilientes y su impacto en la reducción del riesgo de desintegración de las familias de la Fundación Nuestra Señora de Amor. Los objetivos específicos son: (1) Evaluar el efecto antes y después de la implementación de las estrategias en la reducción del riesgo de las familias de la fundación Nuestra Señora del Amor, (2) Analizar la implementación de las estrategias de protección diseñadas para fortalecer las capacidades resilientes de los integrantes de la familia y (3) Analizar el efecto de la resiliencia en la disminución del riesgo social.

Se tomaron algunos referentes conceptuales sobre familias, riesgo y resiliencia familiar. Las familias son un conjunto de personas entre las que median lazos cercanos de sangre, afinidad oadopción, independiente de su cercanía física o geográfica y de su cercanía afectiva o emocional Rubiano y Wortenberg (1991). Una de sus funciones es la protección de sus miembros frente a las amenazas y peligros. Es, asimismo, la unidad básica de la sociedad que contiene un espacio indeterminado que permite a la pareja con los hijos y demás miembros del hogar, crear una minicultura propia y particular.

Las características de la familia Colombiana, hoy por hoy, es producto de los cambios socioculturales, de la violencia y de su impacto sobre la unidad familiar. Esto repercute en la coexistencia de diversidad de tipologías según las teorizadas por Echeverri (2004), correspondientes a la diversidad de regiones, etnias y particularidades actuales, resultado de los ajustes urbanos o rurales y a las condiciones socioeconómicas de los diferentes estratos sociales. 
Ante la presencia de choque en una familia se evidencia la pérdida de valores, sentimientos o de diversos aspectos, que de manera cíclica están presentes, los cuales generan situaciones adversas que pueden ocasionar diferentes manifestaciones de recuperación o disminución de expectativas de manera temporal o permanente, que antecede a una resignificación del propósito y la funcionalidad familiar.

La concepción acerca de los riesgos es un aspecto que no se centra específicamente en las personas y familias de bajo nivel socioeconómico, aunque sí son las más vulnerables a situaciones adversas, pues habitualmente carecen de instrumentos adecuados para manejarlos, siendo proclives a factores de índole social, físicos y culturales que les impiden su desarrollo y progreso tanto económico como afectivo.

De ahí que diferentes autores como Holzmann y Jørgensen (2003), propongan una nueva definición y un nuevo marco conceptual sobre la protección social, cimentada en el manejo social del riesgo, replanteándose desde un marco que incluye tres estrategias para abordarlo: prevención, mitigación y superación de eventos negativos (shocks); tres niveles de formalidad de manejo del riesgo: informal, de mercado y público; y varios actores: personas, hogares, comunidades, ONG, diversos niveles de gobierno y organizaciones internacionales, frente a un contexto de información asimétrica y distintos tipos de riesgo. En esta visión se enfatiza en el doble papel que desempeñan los instrumentos de manejo del riesgo y se promueve la disposición a asumir riesgos.

El proceso de análisis del riesgo se enmarcó desde la propuesta planteada por el Instituto de Educación Preventiva y Atención de Riesgos, A.C., Asociación Civil, destinada al estudio de las adicciones y otros riesgos psicosociales INEPAR (2006, p.30), donde la "la realidad de las situaciones de la vida, pueden y deben variar de localidad a localidad, de cultura a cultura, de persona a persona y son imposibles de medir por su complejidad, pero factibles de ser expresados en metáforas preventivas o de riesgo, que la misma comunidad construye y define".

La resiliencia familiar se refiere a las características, dimensiones y propiedades de las familias que las ayudan a resistir la perturbación ante el cambio y a adaptarse ante las situaciones de crisis. Implica integrar la totalidad de la experiencia en la trama de la identidad individual y familiar como lo proponen McCubbin, H.I. y Patterson, J.M. (1983).

Así la resiliencia describe una buena adaptación en las tareas del desarrollo de una persona, como resultado de la interacción entre el sujeto y la adversidad del medio o un entorno de riesgo constante. Desde esta perspectiva la resiliencia es resultado del proceso que resulta a través de las redes de apoyo y la interacción con los grupos sociales que actuarían como generadores de resiliencia en los individuos.

Estos aspectos, se conjugan y refuerzan las posibilidades de apoyar a las personas como seres humanos íntegros, seguros y capaces de salir delante. Igualmente son promovidos o facilitados por el ambiente, las personas, las instituciones y las familias que intervienen en la interacción de los grupos e individuos que están en situación de riesgo y vulnerabilidad. 
En esta misma línea para Moss, Ramakrishnan, Storms, Siegle (2006), la resiliencia es un proceso construido fundamentalmente desde la familia, quien actúa como soporte para generar esperanza por superar la situación adversa.

\section{METODOLOGÍA}

Enfoque: cualitativo de investigación que procura representar el sentido que las personas dan a sus actos, a sus ideas, y al mundo que les rodea de manera subjetiva.

Tipo de investigación: el tipo de nvestigación seleccionada fue la investigación aplicada que permite mantener una relación permanente entre aquello que la labor de investigación y los diagnósticos arrojan.

Participantes: el estudio se realizó en la localidad Ciudad Bolívar, en la fundación Nuestra Señora de Amor, y se enfoca en el trabajo con familias de bajo nivel socioeconómico y en situación de mayor riesgo social. El universo poblacional fueron 50 familias de la FNSA que se encontraban en situación de riesgo social con relación a factores físicos, psicológicos y sociales como vivienda precaria, desnutrición, problemas sociales (malas pautas de crianza, problemas emocionales, embarazos tempranos, drogadicción, delincuencia, desempleo) desescolaridad, entre otros.

Técnicas: las técnicas que se utilizaron fueron Historia de vida, Entrevista Semiestructurada, La observación participante, Estudio de caso, Grupo focal. Estas técnicas permitieron una descripción cualitativa de las características sobresalientes de los integrantes de las familias, permitiendo un acercamiento entre la población y los investigadores, desde la cual se realizaron conversaciones informales con los miembros de las familias, logrando identificar antecedentes personales y familiares, para identificar creencias, sentimientos, miedos y posiciones sociales, permitiendo recoger opiniones, ideas y críticas sobre temas de interés.

Instrumentos: los instrumentos que se utilizaron fueron Formato Historia de vida, Formato entrevista y Diarios de campo.

El procedimiento realizado se presenta en varias fases: se parte de un diagnóstico del riesgo, de los factores resilientes y de la funcionalidad familiar en que se ubicaban las familias. Posteriormente se presenta la recolección de datos, a partir de las entrevistas, historias de vida y actividades de socialización que permitían mayor reconocimiento de la familia. Luego estos datos fueron clasificados, a partir de categorías inductivas y según las categorías deductivas que iban emergiendo. Lo que permitió dar un primer avance de la situación pre de los grupos familiares y sirvió de insumo para el diseño y elaboración de las estrategias de fortalecimiento de los factores resilientes. Se realizó la evaluación de la implementación de las estrategias fundamentadas desde los esos factores y su relación con la disminución del riesgo en cada una de las familias. Los resultados se obtuvieron a partir de la interrelación que se recoge de la implementación de las estrategias de fortalecimiento de los factores resilientes en 
correspondencia con la disminución del riesgo y su manera de afrontarlo. (Ver figura. 1) En ella se muestra el procedimiento que se realizó para llevar a cabo la investigación.

Figura 1. Procedimiento realizado en la investigación

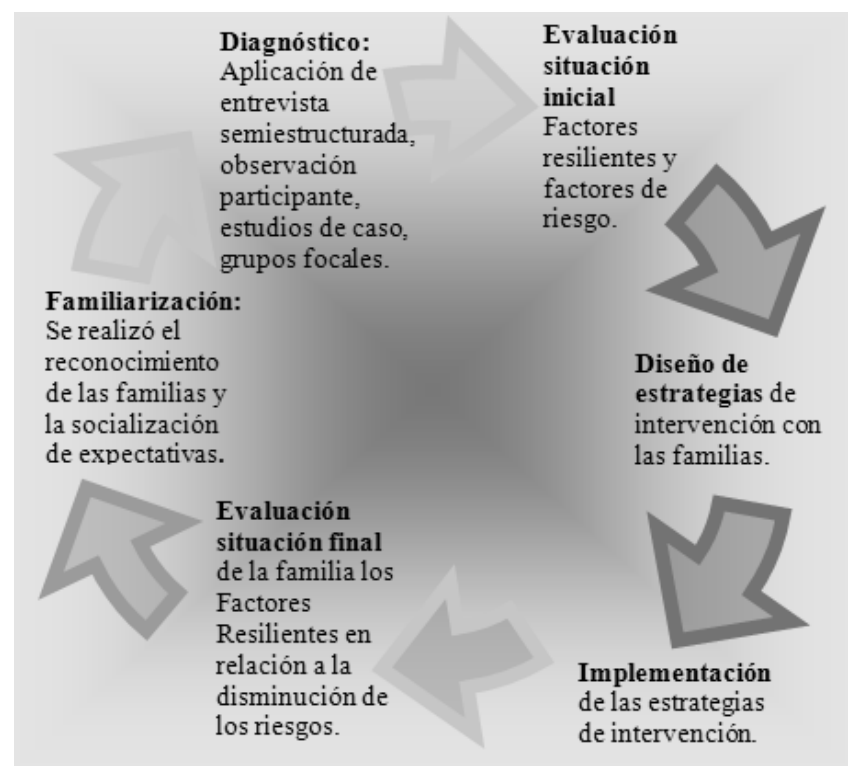

\section{RESULTADOS}

\section{Diagnóstico}

\section{Evaluación del riesgo social en las familias con la Metáfora del semáforo}

A partir de las entrevistas, estudios de caso y observaciones participantes se diseñó, bajo la técnica del semáforo, una tabla en la que se puede evidenciar el riesgo de ruptura del sistema familiar.

En primera medida se presenta la evaluación del riesgo de mayor incidencia dentro de los grupos familiares así como su vulnerabilidad, este proceso se realizó a nivel de cada familia, lo que permitió evidenciar cuáles de ellas estaban más expuestas al alto riesgo y qué aspectos se convierten en factores protectores para las de menor riesgo en un mismo ambiente social.

A continuación se presentan los resultados de la evaluación del riesgo social que se hizo con cada una de las familias. (Ver tabla. 1). 
REVISTA DE INVESTIGACIONES UNAD

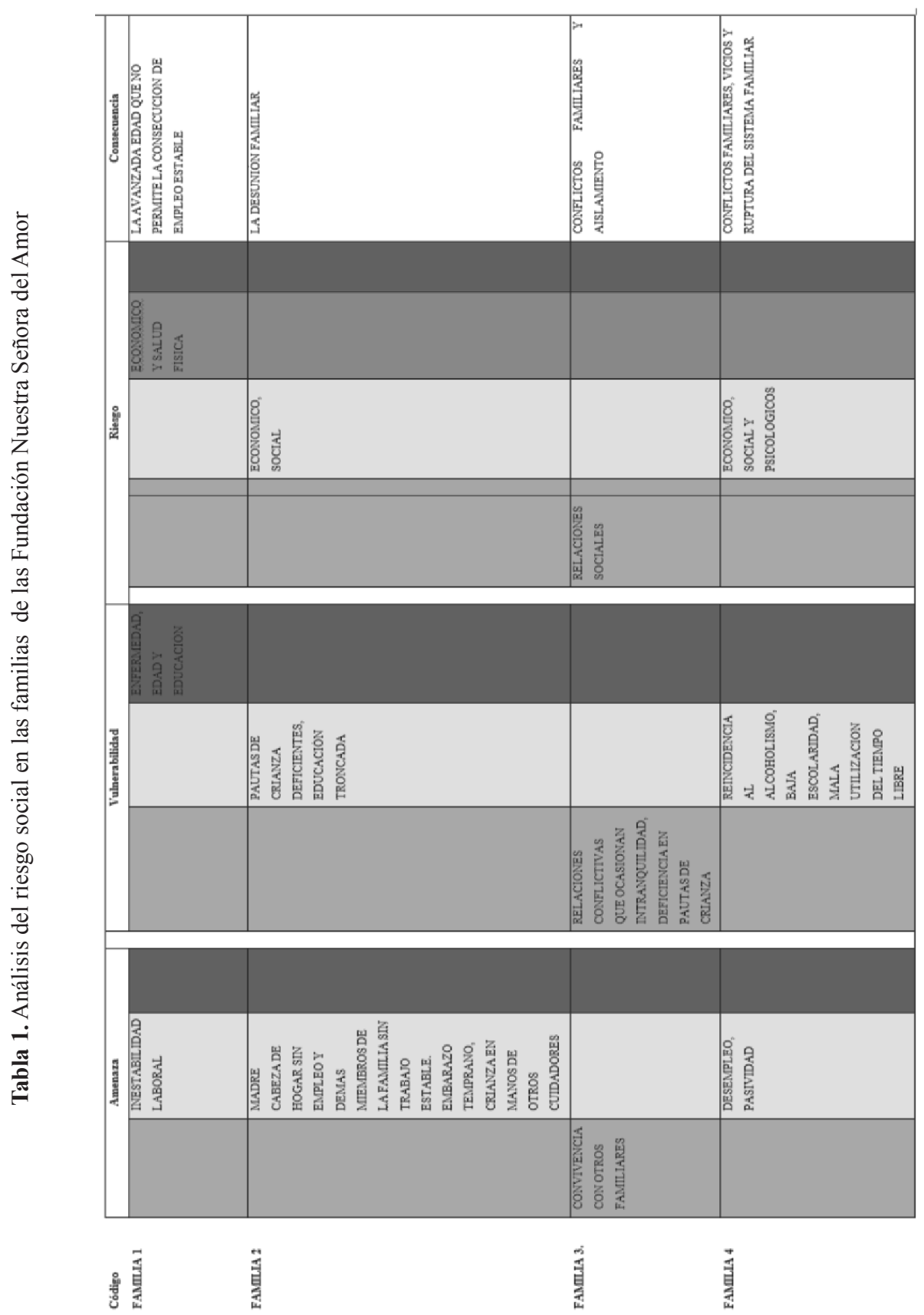


La Tabla 1 refleja las diferentes situaciones de amenaza, vulnerabilidad y de riesgo de las familias de la FNSA, evidenciando que el mayor riesgo al que están expuestas, se relaciona con el factor económico, seguido por las relaciones sociales y lo psicológico. Esto evidencia que el factor económico es un factor que juega un papel importante, pero que se encuentra aliado a cuestiones personales y de interrelaciones que se deben mejorar para fortalecer los vínculos afectivos y de convivencia. Los cuales al no ser manejados de una manera activa y propia, pueden generar un ambiente difuso e inapropiado para la formación completa de cada integrante de la familia.

A continuación se muestran algunas figuras que representan ciertas categorías de análisis recogidas de los instrumentos aplicados a las familias de las FNSA, en relación con aspectos personales y de relaciones sociales y familiares.

La figura 1. Antecedentes de la formación de la familia

\begin{tabular}{|l|l|}
\hline $\begin{array}{l}\text { Amor a primera } \\
\text { vista }\end{array}$ & 3 \\
\hline Romance & 3 \\
\hline Conquista & \\
\hline Relación & 3 \\
\hline Noviazgo & 4 \\
\hline Amistad & 4 \\
\hline \multicolumn{2}{|c}{} \\
\hline \multicolumn{2}{|c|}{} \\
Se conforma
\end{tabular}

desde el

\begin{tabular}{|l|c|}
\hline Hogar & 4 \\
\hline Matrimonio \\
Hijos \\
Solución a problemas \\
Lucha \\
Mejora de la calidad de vida \\
del hogar \\
\hline
\end{tabular}

con el
Momentos tristes

Enfermedades

Embarazos tempranos de hijas

Fallecimiento de padres

Conflictos familiares

Perdidas materiales

Malos negocios

Cambios en las formas de pensar

Separaciones

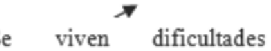

emocionales y económicas

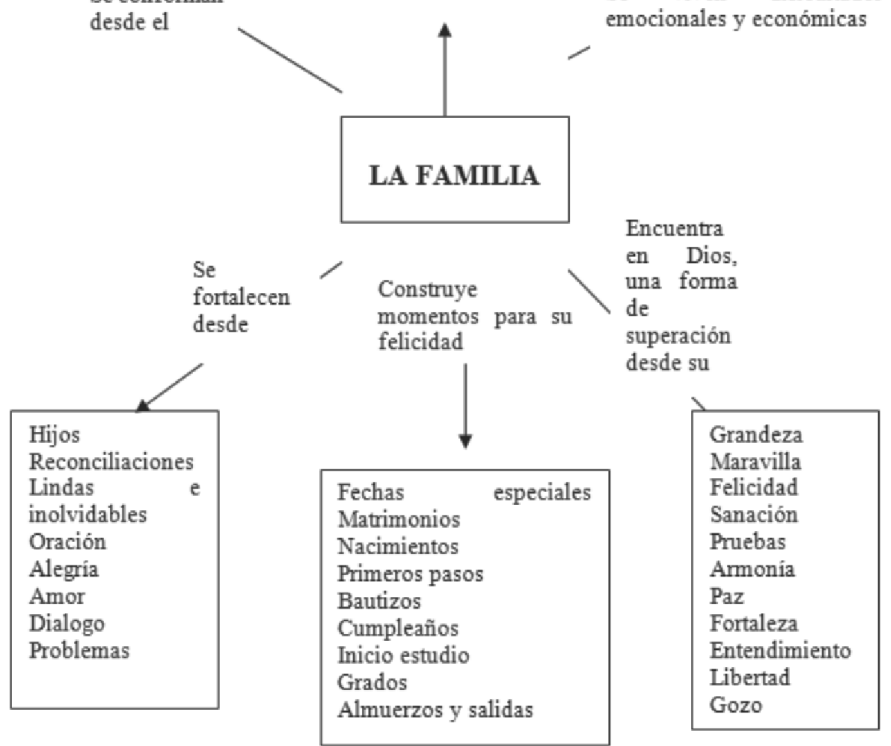


La figura 1. Presenta los antecedentes de cómo se conformaron las familias desde una mirada reflexiva de las diferentes etapas y procesos por las que pasaron y hasta la actualidad.

Se encontraron aspectos que recogen la definición de familia desde la misma comunidad, rescatándose los momentos significativos, las dificultades de mayor incidencia y el valor de la espiritualidad como punto de partida para la superación de problemas. En complemento, este proceso familiar se dio en una línea de vivencias positivas y negativas, y en relación con situaciones que se presentan, que pueden ser debilitadoras e integradores de valores y experiencias que fortalecen y transforman.

Figura 2. La familia desde la comunidad
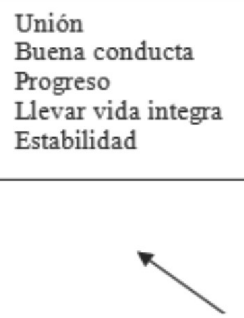

Tiene como Misión la

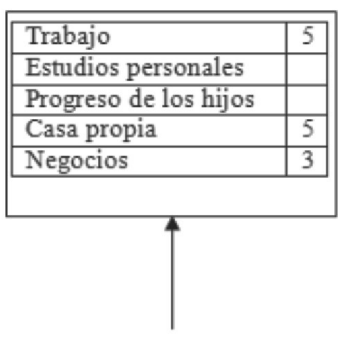

Se construye desde unos sueños $y$ esfuerzos

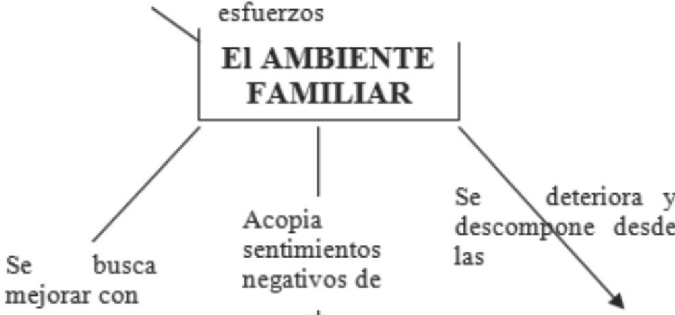

Nuevas religiones Cambio actitudes Apoyo amigos Separación positiva $y$ en acuerdo Abandono vicios (alcoholismodrogadicción)

\begin{tabular}{||l|l|}
\hline Tristeza & 3 \\
\hline Soledad & 3 \\
\hline Dolor & \\
\hline $\begin{array}{l}\text { Impacto } \\
\text { emocional }\end{array}$ & \\
\hline Malos recuerdos & \\
\hline Distanciamiento & 3 \\
\hline $\begin{array}{l}\text { Frustración } \\
\text { personal }\end{array}$ & 4 \\
\hline
\end{tabular}

\begin{tabular}{||l|l||}
\hline Buenas relaciones & \\
\hline Instrucción & \\
\hline Valores & \\
\hline Orientación & \\
\hline Guía & 3 \\
\hline Educación & 5 \\
\hline Corrección & 3 \\
\hline Herramientas & \\
\hline
\end{tabular}

Otorga a los hijos

\begin{tabular}{|l|c|}
\hline Discusiones & \\
\hline Peleas & 3 \\
\hline Celos & \\
\hline Abortos & \\
\hline Miedo & \\
\hline Desempleo & \\
\hline Maltrato verbal & \\
\hline Diferencias de edad & \\
\hline Alcoholismo cónyuges & 4 \\
\hline Drogadicción hijos & \\
\hline Fiestas constantes & 3 \\
\hline Embarazosinesperados & 5 \\
\hline
\end{tabular}


Figura 3. La familia y sus características ambientales

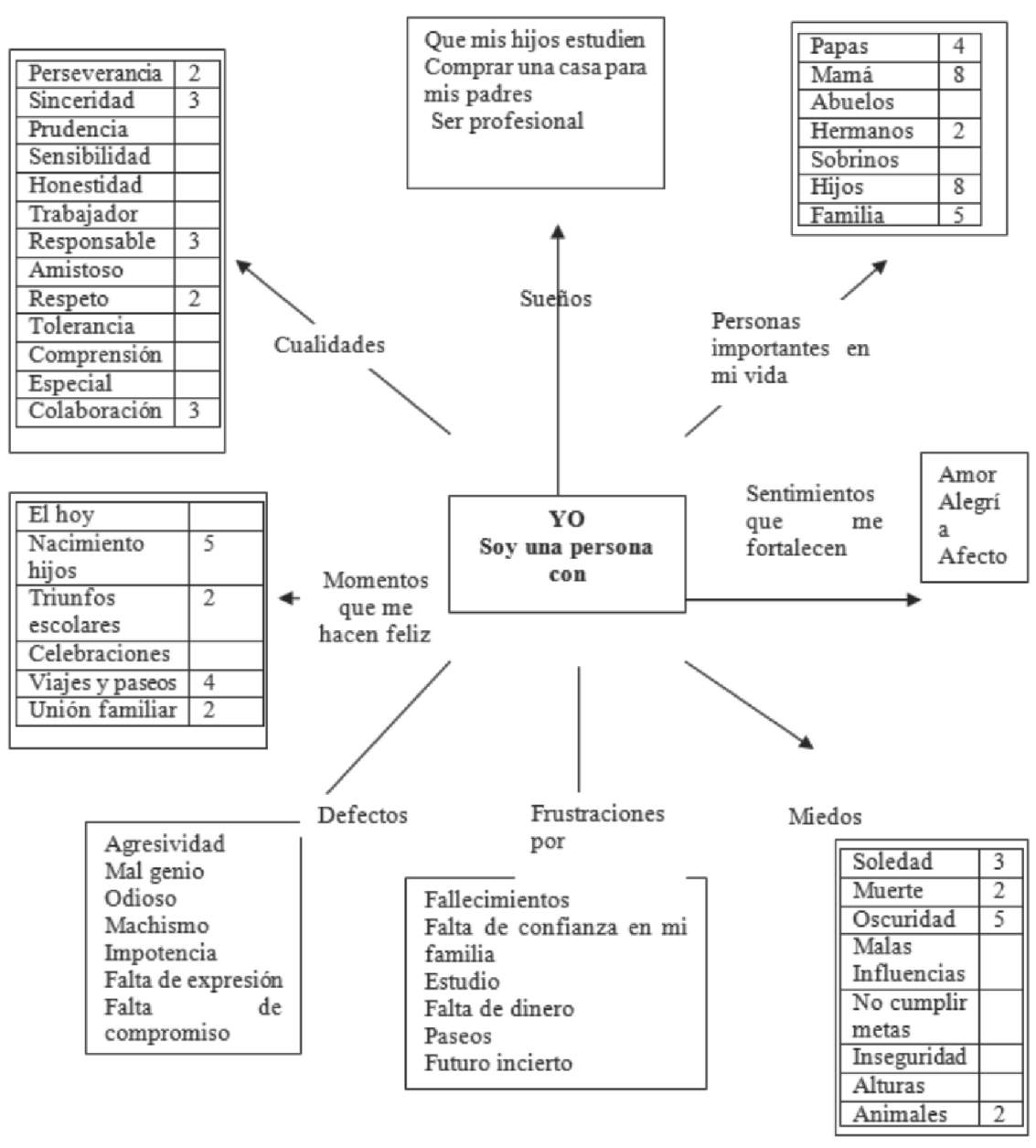

Presentan las características ambientales, en cuanto a la fundamentación psicosocial y a la funcionalidad del grupo familiar, en la constitución de un espacio adecuado para la comunicación, la solución de conflictos y la construcción colectiva de proyectos de vida.

Figura 3. Presenta la percepción personal y proyectiva que tienen los diferentes miembros de las familias, sobre sí mismos para el cumplimiento de sus metas y como estas se convierten en el motor para trabajar día a día por sus aspiraciones y el progreso familiar, además la importancia de reconocer las habilidades y capacidades con las que contaban y podían fortalecerse. 


\section{Resultados del diseño de Estrategias con la comunidad}

El diseño de estrategias para la intervención y disminución del riesgo en la familia fue un proceso dinámico y creativo en donde se tuvieron en cuenta las necesidades emocionales, familiares y entraron en juego las expectativas que tenían las personas involucradas en el proyecto.

La construcción de cada estrategia se elaboraba respondiendo a las expectativas y necesidades observadas y manifestadas por ellos. Además de las falencias encontradas, las acciones se tomaban en el transcurso de los encuentros donde se descubrían diferentes aspectos como actitudes, aptitudes y ambientes que permitían el trabajo de diferentes temáticas de interés y de crecimiento, además de recalcar la importancia de aspiraciones, sueños y metas, en busca de consolidar su proyecto de vida.

La evaluación de los factores resilientes inicialmente, sirvió como guía para trabajar los factores ausentes, vinculándolos al fortalecimiento de uno o varios de estos.

Impacto de la Aplicación de las Estrategias en Relación al riesgo, los Factores Resilientes y la Funcionalidad Familiar

Figura 4. Resultados de implementación de las estrategias de fortalecimiento

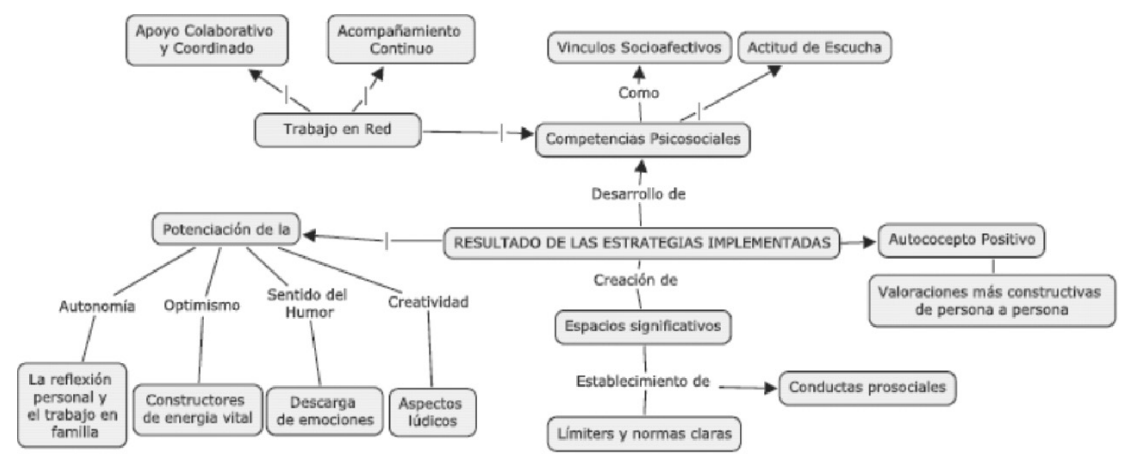

La figura 4. representa el efecto de las estrategias resilientes en el afrontamiento activo y propositivo en las situaciones que se les presentaban al interior de sus hogares, que aunque eran difíciles de cambiar podían ser atenuadas y así lograr un cambio en las actitudes pasivas de cada uno de sus integrantes.

Se orientó en el desarrollo de competencias psicosociales aprendidas en la misma acción social, durante los encuentros que se dieron con la población, logrando con esto un análisis de diversos factores que acompañan la socialización familiar, estos fueron: el acompañamiento continuo, los vínculos socio-afectivos y la actitud de escucha, que se observaron en situaciones en las cuales las personas fueron generadoras de ambientes y fuentes de apoyo social para otros, al dedicar tiempo y espacios a escuchar y hacer esfuerzos sinceros por entender antes de 
juzgar o mejor no juzgar. Estableciendo relaciones de confianza y afecto entre los mismos. Creación de espacios que facilitaron el encuentro al interior de los grupos familiares, que en ocasiones eran distantes y tensos por diferentes razones, dentro de estos se establecían límites y normas claros que permitían que las familias se apropiaran y fomentaran emociones amorosas, conductas pro sociales y actitudes más positivas.

El auto-concepto positivo es el resultado de que los padres aprendieron a valorar a sus hijos por las apreciaciones que de ellos reciben de personas externas, siendo esta interrelación padre e hijo lo que fortaleció el concepto que tenían de sí mismos, el auto-concepto y dio una valoración más constructiva de persona a persona, ayudándoles a ver los aspectos positivos de la familia e incorporando nuevas actividades como elementos resilientes.

Potenciación de la autonomía a través del valor de la responsabilidad: se evidenció que en las familias es donde se deben establecer y fundamentar los compromisos a los hijos, lo que permitió en muchas de las familias establecer una forma correcta de enseñanza, contrario de las que cayeron en el error de que los hijos se volvieran dependientes y no tomaran decisiones propias. En el sentido que si se les proveían todo o se les decía siempre lo que había que hacer decidiendo por ellos, lo que se conseguía eran comportamientos más correctos pero no se facilitaba el aprendizaje de la autonomía. La anterior situación resalta que el aprendizaje de los valores es un escenario que se logra desde la reflexión personal y el trabajo en familia, y que involucra cambios en la idea y forma de ensenar y aprender de los otros.

Creación de espacios para hablar de la propia vida: hoy, como siempre, las personas necesitan compartir ideas, sentimientos y experiencias con la gente de su edad y también con profesionales comprometidos y capaces de escuchar. Como resultado de esto se encontró que estas actividades en grupo permitían hablar de sus amistades, de la misma familia y además de otros aspectos de interés. Esto se convirtió en un modelo a seguir ya que lo vivido en los grupos de trabajo se llevaba a compartir experiencias dentro la familia, promoviendo los hábitos de comunicación tan vulnerables en las relaciones de familia.

En relación con el optimismo y el sentido del humor, estas expresiones se evidencian como factores constructores de energía vital ante las dificultades, hallando que en las familias, los momentos de diversión permitieron descargar emociones y aliviar situaciones que ocasionaban malestar psicológico y físico. A través de la creatividad, se da rienda suelta a la dimensión lúdica que ayuda a relativizar las situaciones difíciles de la vida. El baile, la música, la pintura facilitó la expresión de emociones y la creación de ambientes propicios para desarrollar la creatividad, además de disfrutar con los otros, compartir y reírse.

Trabajo en red: se idealiza en el trabajo coordinado entre los miembros de la familia. Este trabajo se generó desde el apoyo colaborativo entre los mismos y posteriormente se trasmitió a las demás familias de la fundación. Así, se convirtió en un proceso que facilitó el trabajo y la consecución de actividades en conjunto como fue la planeación de proyectos productivos, salidas recreativas y de acompañamiento colectivo a las familias con mayores problemas, haciendo más fácil la elaboración de soluciones. El ejercicio de las redes familiares, es de 
gran impacto en relación con el trabajo con familias que se vuelven las gestoras principales de la transmisión y la vivencia de la resiliencia.

El trabajo en equipo y el ejercicio de la comunicación se evidenció a partir de las diferentes actividades que implicaban la facilidad de expresión verbal, en donde se generaban comportamientos de seguridad y confianza en los miembros de la familia, lo que conformó modelos a seguir frente a la mejora de las relaciones humanas.

Por último, estas estrategias formaron parte de un escenario social que se orientó hacia el fortalecimiento de la familia, en la consolidación y unión de esfuerzos de los pequeños grupos de la comunidad, creando vínculos y proporcionando a la familia una base segura para enfrentarse a obstáculos y reforzar creencias, tradiciones y factores resilientes que le lograron dar un nuevo sentido y le permitieron ubicarse de manera activa y participativa.

\section{DISCUSIÓN}

Bajo la mirada realizada en esta investigación se llega a comprender la incidente relación entre riesgo social en la familia y la resiliencia como estrategia de prevención, y proceso estratégico para la mitigación de los problemas de la sociedad. Los resultados confirman que la familia es una de las instituciones más importantes de la sociedad y se consolida desde todos los procesos básicos del ser humano, a partir de escenarios que la conforman y le permiten ser modelo de comportamiento para sus integrantes en diferentes situaciones. La familia es creadora de ajustes, incentivadora de cambios y de un núcleo social resiliente, que reúne todos los elementos y los aportes conceptuales que se dan en relación con la resiliencia en familia y que fueron el sustento de las estrategias aplicadas, afirmando que ésta sólo se da en interacción con grupos sociales y desde la autorrealización creativa según el ambiente en el que se ha formado el hombre. Además, este proceso le permite consolidarse como una institución equilibrada para el manejo de cada necesidad, que pueda darse desde el cuidado afectivo del niño como parte fundamental para la formación del adulto significativo.

Se halló que cada persona por su edad, su condición física, su educación y su estado socioeconómico, además de sus antecedentes familiares, puede presentar diversidad de características, como lo menciona (Leibovich et al., 2007), esto hace de la familia un escenario complejo que reúne una serie de situaciones, costumbres, conocimientos y riesgos, que a partir de aspectos individuales tienden a involucrar a toda la familia, es decir, es el epicentro que reúne las cualidades, fortalezas, debilidades y amenazas de sus miembros, mostrándola en un ámbito diverso y cosechador en los momentos de generación de cambio.

De manera específica, se resalta cómo desde las pautas de crianza, dadas por los progenitores (madre cabeza de hogar, acudientes u otros familiares) se evidencia una pérdida en la formación de valores y normas para un adecuado proceso evolutivo saludable que pueda minimizar los efectos de exposición del individuo a los factores de riesgo: como madres adolescentes, drogadicción, delincuencia, problemas emocionales, entre otras, que llevan a la desintegración familiar y por consiguiente a conflictos y malas relaciones interpersonales, 
sin dejar de lado la condición económica por causa del desempleo y la escaza educación por la que pasan estas familias de la FNSA. Frente a los resultados se evidencia que aunque no se lograron cubrir en su totalidad estas deficiencias, si se lograron mejorar la relaciones de padres e hijos, y la implementación de estrategias creativas para minimizar los riesgos a los que estaban expuestos por la mala crianza, esto se ve aplicado en los resultados de las estrategias de fortalecimiento de los factores resilientes y al vinculo familiar.

Se encuentra que el vínculo familiar es un gran influyente en el desarrollo socio-afectivo y cognoscitivo de cada uno los miembros de las familias, especialmente en el de los niños, donde la interacción y las relaciones de apego entre madre, padre y el niño promueve ciertos mecanismos sicológicos protectores, ante situaciones de pobreza y demás problemáticas.

En este sentido, el vínculo que se establece en las familias, a través de las estrategias de interacción y fortalecimiento de los factores resilientes son un indicador para su autodesarrollo, porque les permitió reformular las situaciones de crisis en conjunto con su familia, desde sus mismas capacidades y recursos. Con el cambio personal que se obtuvo en los integrantes de cada familia, se observa la disminución de factores negativos que llevan al fracaso y a múltiples problemáticas, como lo menciona Muños y Graña (2001), la disminución de estos genera herramientas para obtener un entorno tranquilo y armonioso dentro del núcleo familiar.

El proceso educativo es un aspecto central y una estrategia favorable para la superación de la situación económica, siendo un aspecto psicosocial con el que se puede superar la pobreza y dar una mejor mirada a la calidad de vida, además de ser un escenario esencial para la protección y mitigación de los riesgo preexistentes en los niños, los jóvenes y los adultos en la sociedad actual.

Esto revela de alguna manera, que muchos de los problemas que se presentan actualmente en la sociedad, se podrían aminorar desde la organización familiar. Se pueden presentar como propuesta las estrategias de intervención desde la resiliencia, en espacios de recreación y fortalecimiento de los vínculos socio-afectivos, con la aplicación de acciones pertinentes, relevantes y sostenibles a las situaciones de las familias, constituyendo a estos grupos familiares en un modelo de desarrollo y de protección, frente a las amenazas y peligros de cada uno de sus miembros, partiendo de que el cambio se inicia en lo personal y para ello el entorno familiar es el principal medio para fomentar y construir seres humanos íntegros y fuertes en el momento de asumir las adversidades.

\section{REFERENCIAS BIBLIOGRÁFICAS}

ÁLVAREZ, C. Kotliarenco, M.A.; Cáceres, I. (1996), Resiliencia: construyendo en adversidad CEANIM, 119 p. Seminario "Pobreza, Desarrollo Humano e Intervención Social: la Resiliencia como una Posibilidad" organizado por CEANIM. Santiago, Chile.

CASTILLO, Moisés. (2005), Método de estudio de caso. Metodología de investigación USN. http://www.usn.edu.mx/artman/publish/article_16.shtml. 
CEANIM Y FUNDACIÓN VAN LEER. CHILE. ALLEN, V. (1996), The Psychology of Poverty: Problems and Prospects. En: V. Allen (Ed.), Psychological.

BALCÁZAR, Fabricio E. (2003), Investigación Acción Participativa IAP: aspectos Conceptuales y Dificultades de Implementación. Fundamentos en Humanidades, año/ Vol.4 número 007-008. Universidad Nacional San Luis. Argentina-San Luis.

DNP e ICBF. (2000), La demanda de seguridad social de las familias. Biblioteca Luís Ángel Arango. 368.4 D35. Colombia.

ECHEVERRI ÁNGEL, Ligia. (2004), La Familia en Colombia Transformaciones y Prospectiva. Ligia Echeverri Ángel. Cuaderno CES, núm.. 6. CES, Centro de Estudios Sociales, Facultad de Ciencias Humanas, UNC, Universidad Nacional de Colombia, Bogotá, Colombia: Colombia.

GENTILE, S.; Mesurado, B., \& Vignale, P. (2006), Resiliencia en Familias: Una Intervención en contexto educativo en riesgo social. $1^{\circ}$ Congreso Regional sobre Abordajes Comunitarios e Institucionales: Prácticas, Investigaciones e Innovaciones”. Universidad Católica. Argentina. Facultad de Humanidades. Sede Paraná, Entre Ríos.

GOETZ, J.P. y LeCompte, M.D. (1988), Etnografía y diseño cualitativo en investigación educativa. "Evaluación del diseño etnográfico". Madrid. Ediciones Morata, S.A.http://www. monografias.com/trabajos65/observacion-participante/observacion-participante.shtm.

GREEFF Stephanus Abraham. Merwe Van Der. (2004), Variables Associated with Resilience in Divorced Families. Springer Netherlands. Humanidades, Derecho y Ciencias Sociales. Volume 68, Number 1 / agosto.

HERNÁNDEZ C. Ángela. (1998), Familia, Ciclo Vital y Psicoterapia Sistémica Breve. Editorial El Búho. Bogotá.

HERNÁNDEZ MARTÍNEZ, H. y González Sierra M. (2003), Extensión de la Cultura ¿Tarea Olvidada? Seminarios Locales. Comisión Especial para el Congreso Universitario. CECU-UNAM. Consultado el 22 de noviembre de 2006, en: http://www.cecu.unam.mx/ ponsemloc/ponencias/1416.html.

HOLZMANN, Robert y Jørgensen, Oteen. (2003), Manejo Social del Riesgo: un nuevo marco conceptual para la protección social y mas allá. Este documento es una versión revisada, durante la preparación del Documento de Estrategias para el Departamento de la Protección Social. Revista de la Facultad Nacional de Salud Pública, enero-junio, vol. 21, número 1. Universidad de Antioquia Pág. 73-1. Colombia.

HOMBRADOS, María I. (1996), Introducción a la psicología social comunitaria. Ediciones Aljibe. 
INEPAR. INSTITUTO DE EDUCACIÓN PREVENTIVO Y ATENCIÓN DE RIESGOS. Un instrumento epidemiológico para la medición de conductas de riesgo/protección psicosocial en poblaciones de jóvenes y adolescentes. (2005). Website: http://www. inepar.edu.mx. México.

JADUE. J, Gladys. (2003), Transformaciones familiares en Chile: riesgo creciente para el desarrollo emocional, psicosocial y la educación de los hijos. Estudios pedagógicos. [Online]. no.29 [citado 26 Enero 2009], p.115-126. Disponible en la www: $<$ http:// mingaonline.uach.cl/scielo.php

KlEVENS, Joanne. (2001), Violencia física contra la mujer en Santafé de Bogotá: prevalencia y factores asociados. Revista Panamericana de Salud Pública. Revista Panamá Salud Publica vol.9 núm. 2 Washington Feb.

KOTLIARENCO, M.A. (2009), Memorias. I Encuentro de Investigación de Experiencias en Resiliencia. UNAD. Bogotá.

LANDAZÁBAL y otros. (2007), Una luz que brilla: la resiliencia. Análisis de investigaciones realizadas en la última década. Semillero fénix ave de luz. UNAD. Bogotá. Colombia.

LEIBOVICH de F. Marconi, N., Minichiell, A., (2007), Universidad de Buenos Aires (Argentina) Ciencias Psicológicas; I (1): 7-13. Prensa Médica Latinoamericana.

LÓPEZ P. Judith (2006), Resiliencia Familiar ante el duelo. Universidad Nacional Autónoma de México. Facultad de Psicología.

LÓPEZ, A. (1996), La resiliencia algo a promover. Ginebra. En red www.comminit.com /la/ teoriasdecambio/ lacth/lasld-285.html. Recuperado 9 de octubre de 2005.

LÓPEZ, Ibor. (1992), Psicología práctica. La adolescencia. Argentina.

MARTINS, Maira. Dos S. Antonio, Pillon, Sandra C. (2008), Low-income families' perceptions on the use of drugs by one of their members. Revista Latinoamericana Enfermagem. 16(2). www.eerp.usp.br/rlae

MARTÍNEZ, Jeannette. (2007), Guías de trabajo. Métodos y fundamentos de trabajo de campo 3. UNAD. Bogotá.

McCUBBIN, H.I. y Patterson, J.M. (1983), The Family Stress Process: the Double ABCX Model of Adjustment and Adaptation. En H.I. McCubbin, M.B. Sussman y J.M. Patterson (eds.): Social Stress and the Family (7-37). New York: Haworth.

MONTERO, B. Maritza. (1980), Más allá del dilema de los métodos. Venezuela. 
MOSS,W. Meenakshi Ramakrishnan, Dory Storms, Anne Henderson Siegle. (2006), World Health Organization. Bulletin of the World health organization Tomo $84 \mathrm{n} .{ }^{\circ} 1 \mathrm{pg} .58,7$ pgs. Ginebra.

MUÑOZ-R, Marina J. \& Graña L, José L. (2001), Factores familiares de riesgo y de protección para el consumo de drogas en adolescentes. Universidad Complutense de Madrid. Psicothema. Vol. 13, n. ${ }^{\circ}$ 1, pp. 87-94. 2001 Psicothema.

NAVEILlAN, Pedro (2000), Familia y Riesgo Social. Revista El morrocotudo. Primera Parte. http.www.elmorrocotudo.cl. Chile.

PUJADADAS MUÑOZ, Joan J. (2002), El método biográfico: el uso de las historias de vida en las ciencias sociales. Centro Investigaciones Sociológicas Madrid.

RUBIANO, Norma y Wartenberg, Lucy. (2006), Derecho Humanos y derechos de la mujer: ante todo los principios de protección. El Aborto Inducido en Colombia: Características demográficas y socioculturales. http://www.unal.edu.co/bioetica/documentos/ conveniodoc/c1_pr

SANABRIA T. Hugo. (2008), Curso Académico: Intervención Psicosocial en Comunidad. Programa Psicología. Escuela de Ciencias Sociales, Artes y Humanidades. Bogotá, D. C., agosto.

SPENCE A, Pomonis. (2005), Vulnerabilidad y evaluación de riesgo. Coburn. Cambridge Architectural Research Limited. The Oast House, Maling Lane, Cambridge, U.K. 1a Edición.

SUÁREZ, Ojeda, N: Kotliarenco, M.A.; Mardones, F.; Melillo, A.; (2000) Buenos Aires, Universidad Nacional de Lanús, Fundación Bernard van Leer. 111 p.

Veeduría social. Vivir en Bogotá (2002), Vulnerabilidad en Bogotá. Edición 8.

BERTAlanfFy, L. V. (1996), Teoría General de los Sistemas. Petrópolis, Vozes. http:// www.alumnos.inf.utfsm.cl/ vpena/ramos/ili260/textos/tgsbertalanffy.pdf

VINSON, J.A. (2002), Children with asthma: Initial development of the Child Resilience Model. Journal of Pediatric Nursing, 28(2), 149-158.

VIZCARRA, María B. L; Cortés M; Bustos M larcón E; Muñoz N. (2001), Revista médica de Chile V. 129 núm. 12 Santiago dic. Temuco Chile. 